\title{
INFLUENCIA DEL DIÁMETRO MEDIO DEL RODAL Y LAS DISTANCIAS MEDIAS DE EXTRACCIÓN EN LOS COSTOS DE UN SISTEMA DE COSECHA EN BOSQUES DE EUCALYPTUS GLOBULUS EN LA ZONA CENTRAL DE CHILE ${ }^{1}$
}

Palabras clave: Simulación dinámica, volteo, extracción, astillado, costos de producción, Eucalyptus globulus.

Key Words: Dynamic simulation, felling, skidding, chipping, production costs, Eucalyptus globulus.
Alonso Barrios ${ }^{2}$

Ana M. López ${ }^{3}$

Víctor M. Nieto ${ }^{4}$

\section{RESUMEN}

Se estudió un sistema de cosecha de árbol entero y astillado en bosque a través del modelamiento y la simulación dinámica. La plataforma iThink fue utilizada para construir una aplicación que simulara el funcionamiento del sistema de cosecha, utilizando modelos determinísticos y estocásticos (Isee systems, Inc. 2007). Las variables utilizadas en este estudio fueron el diámetro medio a la altura del pecho y la distancia de extracción media del rodal. De esta manera, se determinó la influencia de éstas en los costos por metro cúbico del sistema. Este estudio representa una aproximación técnica para establecer tarifas de pago más apropiadas, teniendo en cuenta que el costo de cosecha varía de acuerdo con las características del rodal.

\begin{abstract}
A whole tree and in-wood chipping harvesting system was studied by modelling and dynamic simulation. The iThink environment was used to build an application for simulating the operation of the harvesting system, using both deterministic and stochastic models (Isee systems, Inc. 2007). The variables used in this study were the mean diameter at breast height and the mean skidding
\end{abstract}

distance of the stand. In this way, the influence of these variables in the system's cost per cubic meter was determined. This study exhibits a technical approach for establishing more appropriate payment fees, considering that the harvesting costs vary according to the stand characteristics.

\section{INTRODUCCIÓN}

La incursión del sector forestal en el mercado internacional ha llevado a las empresas forestales a encaminarse en un proceso de mejoramiento continuo, enmarcado en el concepto de la competitividad. Dentro de este proceso la cosecha forestal juega un rol importante, ya que representa aproximadamente un $40 \%$ de los costos de producción en el sector forestal. En este sentido, lo que se busca es que los sistemas de cosecha aumenten su productividad para que satisfagan la demanda de materia prima de los centros de producción a un bajo costo.

Para cumplir con estas expectativas es preciso diseñar alternativas o estrategias para controlar el costo y la productividad de los sistemas de cosecha. De esta forma, es necesario partir por analizar las relaciones existentes entre los mecanismos dispuestos para la cosecha forestal y las características del

\footnotetext{
1 Estudio realizado a través de una asistencia académica y de investigación en el Instituto de Manejo Forestal de la Facultad de Ciencias Forestales de la Universidad Austral de Chile.

CONIF, alonsobarrios@postgrado.uach.cl

CONIF, analopez@exalumnos.uach.cl

CONIF, victornieto@conif.org.co
} 
rodal sobre el cual van a laborar. Este análisis permite retroalimentar y generar controles al sistema, permitiendo en el corto plazo ser más eficientes. En este proceso, sin duda alguna, los análisis económicos surgen como los más apropiados y de mayor uso en la evaluación de los sistemas de cosecha forestal como los realizados por Provoste (2004), Figueroa (2004), Jaramillo (2005), Arroyo (2005) y Carey et al. (2006).

En la búsqueda del mejoramiento de la productividad y de la asignación correcta de los sistemas de cosecha al rodal con las características acordes que permitan maximizar la producción, el modelamiento matemático y el uso de la simulación han mostrado ser de suma importancia. La simulación en la evaluación de los sistemas de cosecha forestal ha sido aplicada en muchos casos como los de Bradley et al. (1976), Winsauer (1982), Barret (2001), McDonagh (2002) y McDonagh et al. (2004); su principal ventaja radica en la capacidad de replicar infinitamente el mismo fenómeno para facilitar su interpretación y análisis. La capacidad de predecir el comportamiento de un sistema de cosecha que va a actuar expuesto a determinadas características del rodal facilita el proceso de toma de decisiones en cuanto a la elección del sistema más idóneo para tales condiciones (McDonagh et al. 2004).

En el ámbito forestal chileno, la industria pulpable ha venido incrementándose. Muestra de ello es la apertura de nuevas plantas para la obtención de productos celulósicos y la ampliación del patrimonio forestal con especies de rápido crecimiento como Eucalyptus globulus. En este sentido, la incorporación de sistemas de cosecha más productivos es predominante. Entre éstos, los sistemas de astillado en bosque han sido una alternativa de gran importancia para las empresas forestales. Sin embargo, la escasez de información acerca de su productividad y los altos costos de los equipos hacen necesaria la ampliación del conocimiento en este campo.

El objetivo de esta investigación es evaluar económicamente el comportamiento de un sistema de cosecha de árbol entero que adiciona un sistema de astillado en bosques de Eucalyptus globulus en la zona central de Chile en relación con condicionantes externas como el tamaño de los árboles medido a través de su diámetro a la altura del pecho (DAP) y las distancias medias de extracción (DE).

\section{MATERIALES Y MÉTODOS}

\section{INFORMACIÓN UTILIZADA Y ÁREA DE ESTUDIO}

La información del estudio de tiempos y movimientos obtenida por Jaramillo (2005), quien evaluó las características técnicas y económicas del sistema de astillado en un bosque de Eucalyptus globulus en la VIII Región de Chile, se utilizó como base para los análisis presentados en este articulo (Figura 1).

\section{DESCRIPCIÓN DEL SISTEMA DE COSECHA}

El sistema estudiado presenta un alto nivel de mecanización donde se pueden identificar los siguientes subsistemas o actividades:

Volteo: realizado por un feller-buncher marca Tigercat modelo 720C (Figura 2a). El ciclo de trabajo incluyó el corte y volteo del árbol, desplazamiento corto hacia el siguiente árbol en la misma línea de plantación y desplazamiento largo hacia una nueva línea.

Extracción: se realizó utilizando un skidder grapple marca Tigercat modelo 620C (Figura 2b). Los principales elementos del ciclo de trabajo incluyen viaje vacío hasta la ubicación de la carga, cargue, viaje cargado y descargue.

Astillado: realizado por un equipo marca Paterson Pacific modelo DDC-5000G (Figura 2c). Las actividades realizadas por éste incluyen el descortezado, desrame, astillado y carguío de astillas.

Transporte: realizado por un grupo de camiones Freightliner modelo FL112 6x4 (Figura 2d), con motor Mercedes Benz de 410 HP equipados con rampas metálicas diseñadas especialmente para el transporte de astillas. 


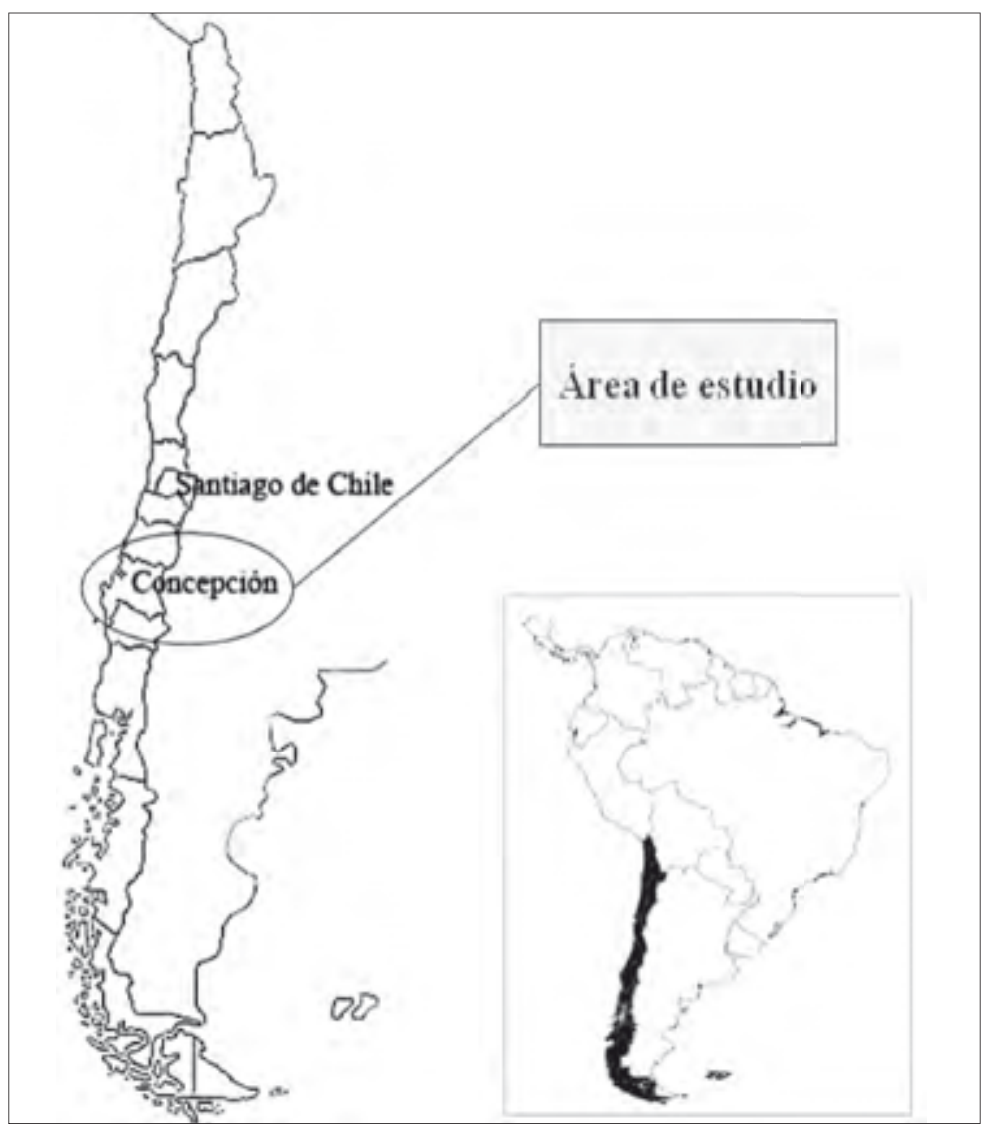

Figura 1. Localización del área de estudio.
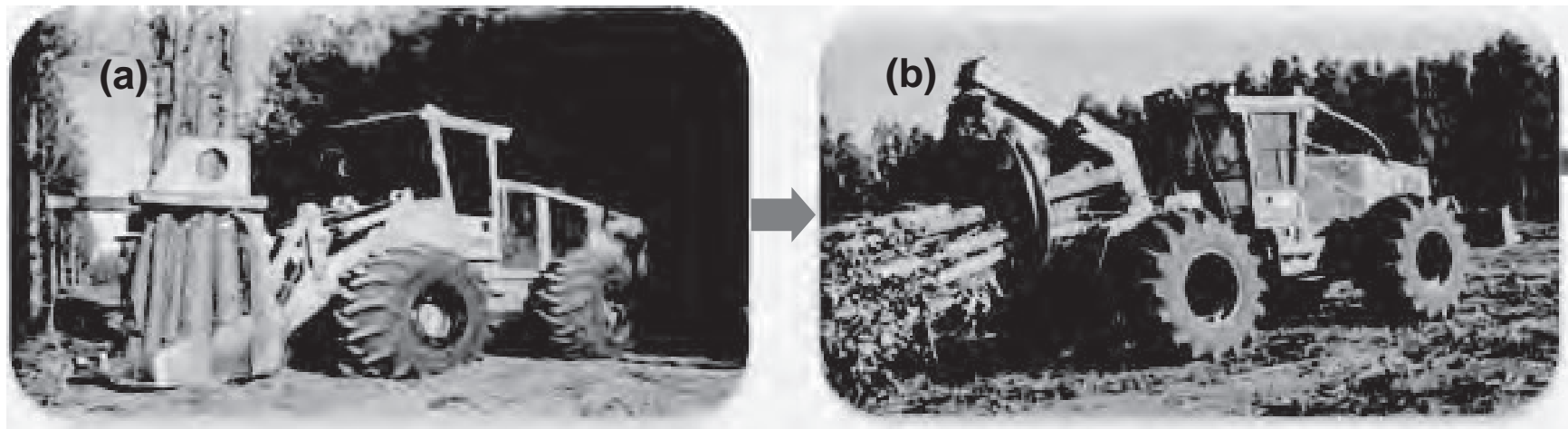

(c)
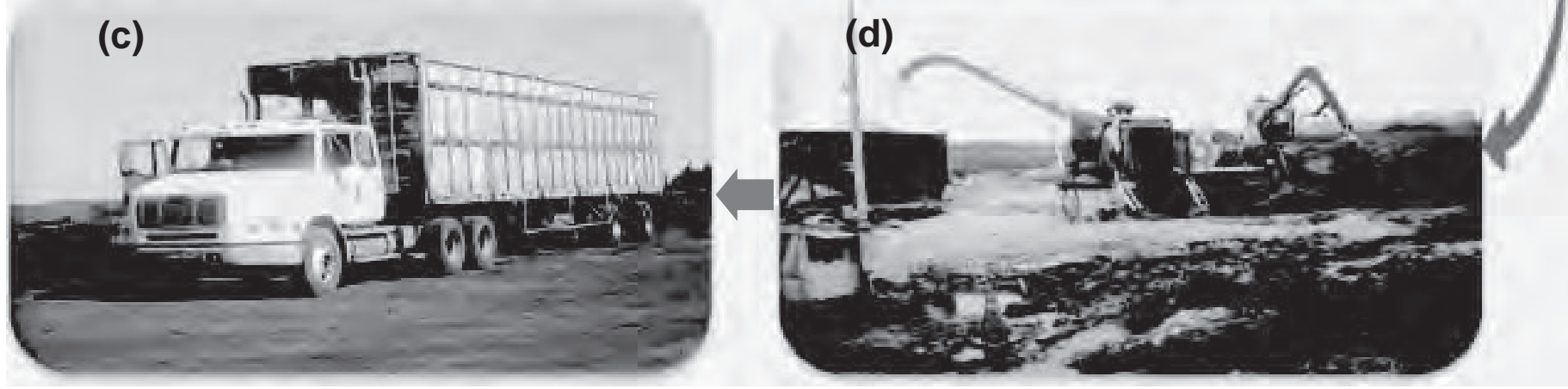

Figura 2. Esquema del sistema de cosecha del estudio. 


\section{MODELAMIENTO Y SIMULACIÓN DEL SISTEMA}

La información de productividad y de demoras obtenida a partir del estudio de tiempos y movimientos se utilizó para construir modelos matemáticos que describieran las relaciones entre las variables de rodal (DAP y DE) y la productividad de cada actividad realizada por el sistema de cosecha. Sólo se evaluó el comportamiento de los subsistemas volteo, extracción y astillado, y el transporte no se consideró en los análisis. La Figura 3 ilustra cómo el sistema de cosecha es influenciado por las variables del rodal y cómo el proceso de simulación aproxima la estimación de la productividad. La máxima productividad de cada subsistema puede ser obtenida cuando las condiciones del rodal no son limitantes, representada por el punto a en la Figura 3. También puede ocurrir que el sistema de cosecha sea asignado a un sitio con condiciones poco favorables, representadas por el punto $b$ (Figura 3), provocando una disminución en la productividad que causará ineficiencias en el sistema. Esta aproximación se utilizó para predecir el comportamiento de la productividad y de los costos del sistema en un rango amplio de condiciones de rodal.

Para la simulación del sistema se diseñó una aplicación utilizando la plataforma iThink. Este software permite la convergencia de modelos determinísticos y estocásticos en sus cálculos. La aplicación fue diseñada para trabajar durante 500 horas, con 60 iteraciones por cada hora simulada, es decir una cada minuto. La elección de la longitud de la simulación tiene gran importancia debido a que al aumentarla se reduce la variación de los resultados (McDonagh et al. 2004).

El modelo de simulación produce valores aleatorios para las variables DAP y DE de manera automática siguiendo una distribución normal, con una media y una desviación estándar. Estos valores son tomados por la aplicación y convertidos en valores de productividad a través de los modelos determinísticos ajustados. Los valores de productividad obtenidos en este proceso son corregidos por la adición del efecto de las demoras de cada subsistema. Las demoras son calculadas a partir de un generador de números aleatorios siguiendo una distribución de probabilidad característica para cada subsistema (McDonagh et al. 2004).

La aplicación utiliza como información de entrada los costos por hora de cada subsistema, discriminados entre costos fijos y costos operacionales. La mano de obra, en este caso, es considerada como un costo fijo. Los costos fijos se producen en cada hora en la que el sistema está planificado para

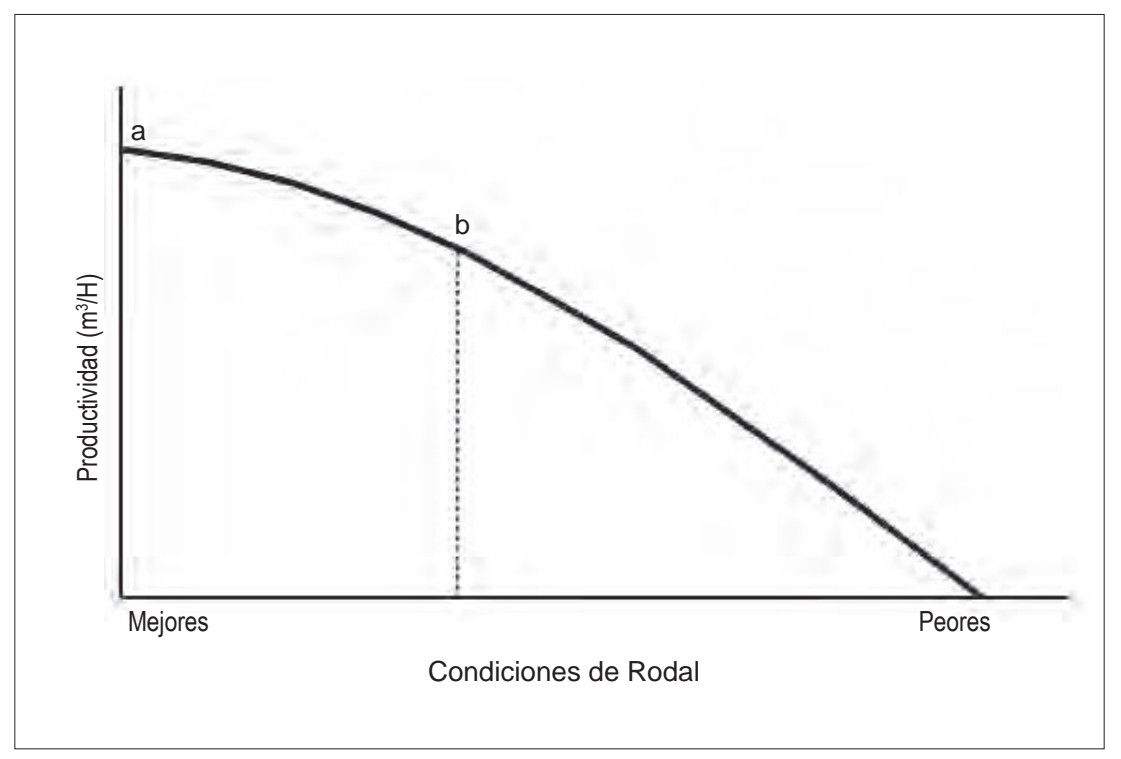

Figura 3. Aproximación al cálculo de la productividad de cada subsistema. 
operar (incluyendo el tiempo de demoras) y los costos operacionales sólo ocurren cuando el sistema está realizando trabajo productivo. Una planilla de cálculo se utilizó para obtener los costos por hora (ver anexo 1).

Influencia del DAP y DE en los costos del sistema. Cada rodal posee características propias que afectan el desempeño técnico de la maquinaria forestal al momento de la ejecución de la cosecha. En forma particular la distancia media de extracción y el tamaño de los árboles definen la eficiencia con que se realizará la operación. La metodología usada simula los diversos escenarios que se enfrentan en la cosecha forestal. Al realizar una variación paramétrica sobre la media de la distribución normal utilizada para la generación de valores de DAP y DE se consigue abarcar un rango amplio de rodales. En el análisis expuesto, la desviación estándar de la distribución normal permaneció fija durante todas las iteraciones. Para el DAP se utilizó una desviación estándar de $4 \mathrm{~cm}$ y para la DE una igual a $100 \mathrm{~m}$.

\section{RESULTADOS}

\section{MODELAMIENTO Y SIMULACIÓN DEL SISTEMA}

Se ajustaron modelos exponenciales utilizando la variable DAP en relación a la productividad para cada uno de los subsistemas. La variable DE sólo se utilizó para ajustar el modelo de productividad para el subsistema de extracción (Tabla 1). Con estos modelos, la aplicación construida calcula la productividad de cada subsistema de acuerdo a los valores de DAP y DE producidos por el generador de números aleatorios.

\section{VALIDACIÓN DEL MODELO DE SIMULACIÓN}

La validación de la plataforma de simulación se llevó a cabo a través de una comparación de medias entre los datos generados por el simulador y los datos originales del estudio de tiempos y movimientos. De esta manera se comprobó que al 95\% de nivel de confianza no existen diferencias significativas entre los valores de productividad $\left(\mathrm{m}^{3} / \mathrm{H}\right)$ y entre los costos de producción del sistema de cosecha $\left(\mathrm{US} \$ / \mathrm{m}^{3}\right)$. Sin embargo, se observa que las mayores concordancias entre la información generada por el simulador y los datos reales se dan en los costos de producción con P-Valores $\geq 0.69$. La Tabla 2 presenta los resultados de este análisis.

\section{EFECTO DE LAS VARIABLES DAP Y DE EN LOS COSTOS DEL SISTEMA}

La evaluación de la influencia de las variables DAP y DE en el sistema de cosecha se realizó utilizando los datos suministrados por la aplicación. Para un rodal con una DE media de 200 metros se realizó una variación sobre la media de la distribución normal que genera los valores del DAP y se obtuvo el comportamiento del costo por metro cúbico del sistema en función de esta variable (Figura 4). La diferencia

Tabla 1. Modelos ajustados para predecir la productividad del sistema de cosecha.

\begin{tabular}{llc}
\hline $\begin{array}{c}\text { Subsistema o } \\
\text { actividad }\end{array}$ & \multicolumn{1}{c}{ Modelo ajustado de productividad $\left(\mathrm{m}^{3} /\right.$ Hora $)$} & R2 \\
\hline Volteo & $\operatorname{Prod}\left(\frac{m^{\mathrm{s}}}{H}\right)=E X P(3,78125+0,0440048 D A P)$ & 0.56 \\
Extracción & $\operatorname{Prod}\left(\frac{m^{\mathrm{s}}}{H}\right)=E X P(1,59131+0,120656 D A P-0,00226959 D E)$ & 0.89 \\
& $\operatorname{Prod}\left(\frac{m^{\mathrm{s}}}{H}\right)=E X P\left(0,105141+0,197861 D A P-0,00264887 D A P^{2}\right)$ & 0.99 \\
\hline
\end{tabular}


Tabla 2. Comparación de medias para la producción por hora y los costos por metro cúbico de cada actividad del sistema de cosecha.

\begin{tabular}{|c|c|c|c|c|c|c|}
\hline \multirow{2}{*}{ Subsistema } & \multicolumn{3}{|c|}{ Producción $\left(m^{3} / H\right)$} & \multicolumn{3}{|c|}{ Costo medio de producción (US\$/m³) } \\
\hline & Observado & Estimado & P-Valor & Observado & Estimado & P-Valor \\
\hline Volteo & 106,50 & 106,69 & 0,91 & 0,68 & 0,65 & 0,77 \\
\hline Extracción & 43,03 & 41,71 & 0,38 & 1,51 & 1,47 & 0,69 \\
\hline Astillado & 22,20 & 21,18 & 0,09 & 6,55 & 6,57 & 0,84 \\
\hline
\end{tabular}

entre las curvas de costo total y costo fijo representa los costos operacionales incurridos por el sistema. La curva de costo total indica que el sistema de cosecha es muy dependiente del diámetro medio del rodal. Cuando el DAP aumenta a valores mayores a $25 \mathrm{~cm}$ el costo por metro cúbico baja a valores por debajo de los US $\$ 8 / \mathrm{m}^{3}$. Al seguir aumentando el DAP medio del rodal no se obtienen cambios substanciales en los costos y se puede decir que el costo por metro cúbico alcanza un estado de equilibrio.

Para un rodal con un diámetro medio igual a $20 \mathrm{~cm}$ se hizo variar la distancia media de extracción y se obtuvo la información de la Figura 5. La distancia de extracción media del rodal también afectó el costo del sistema pero en una menor proporción. La diferencia entre las curvas de costo total y costo fijo en la Figura 5 suministra como resultado los costos operacionales del sistema en el procesamiento de un metro cúbico de madera.

\section{COSTOS TOTALES DEL SISTEMA}

Los costos por metro cúbico totales del sistema son calculados como la suma de los costos parciales de cada subsistema. De esta manera pueden ser segregados y analizados en forma individual. Al establecer un DAP medio de rodal igual a $20 \mathrm{~cm}$ y una DE media igual a $200 \mathrm{~m}$, el costo por metro cúbico del sistema alcanza los US $\$ 10,87 / \mathrm{m}^{3}$, de los cuales $78 \%$ corresponden al funcionamiento del sistema de astillado, 16\% al subsistema de extracción y el restante 6\% al subsistema de volteo (Figura 6a). Separando los costos en fijos y operacionales se observa que los costos fijos del sistema de cosecha y astillado en bosque alcanzan el $26 \%$ de los costos

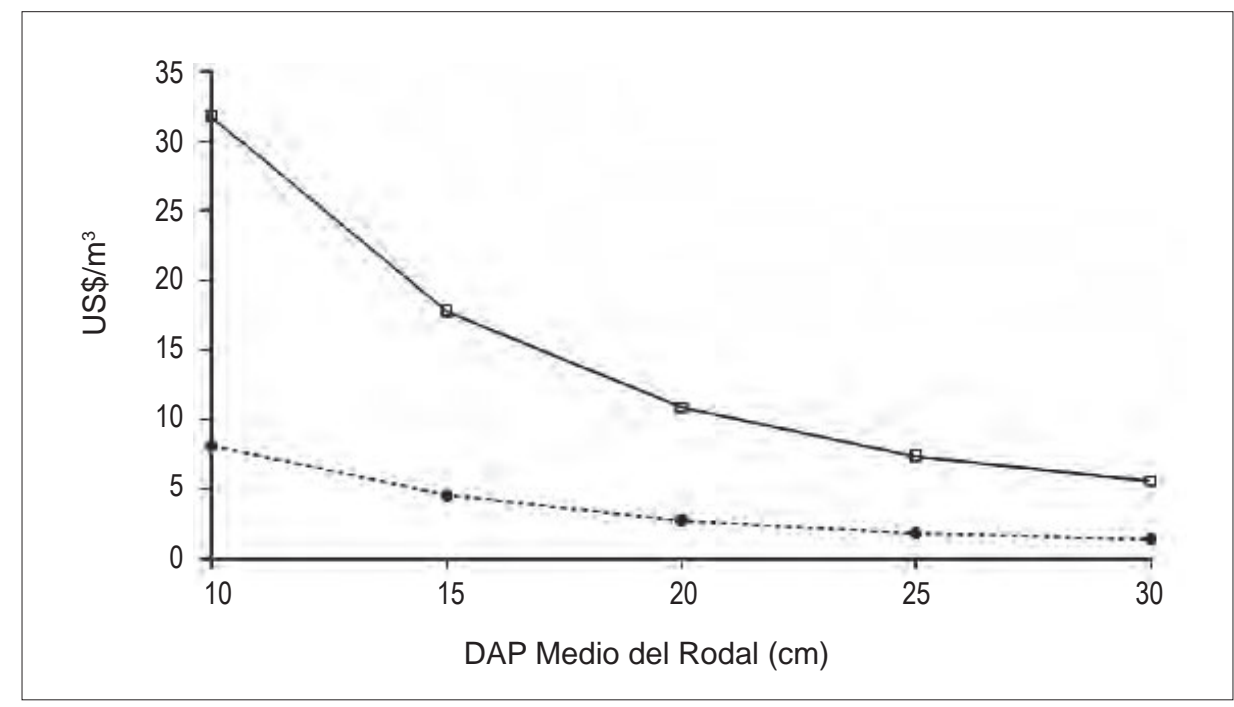

Figura 4. Curvas de costo por metro cúbico del sistema de cosecha en función del DAP medio del rodal. ( $\square-$ ) es el costo por metro cúbico total y $(\cdots \bullet \cdot)$ es la curva de costo fijo del sistema. 


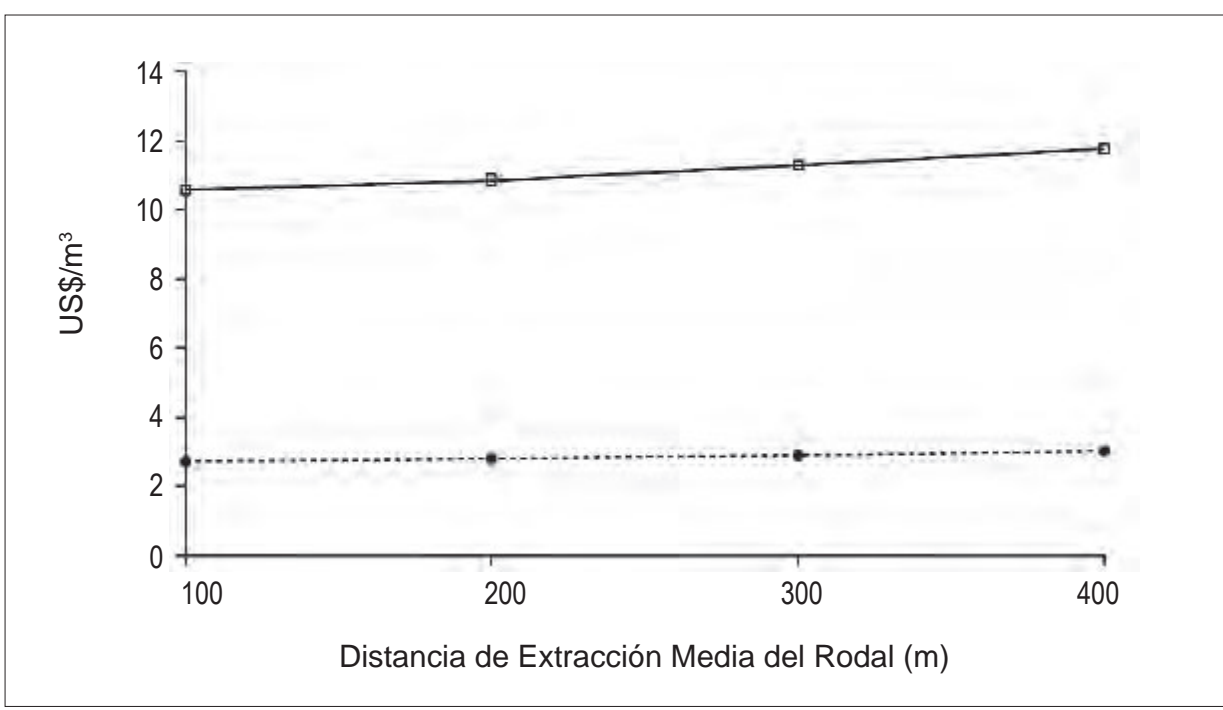

Figura 5. Curvas de costo por metro cúbico del sistema de cosecha en función de la DE media del rodal.( $\square$ ) es el costo por metro cúbico total y $(\cdots \bullet \cdots)$ es la curva de costo fijo del sistema.

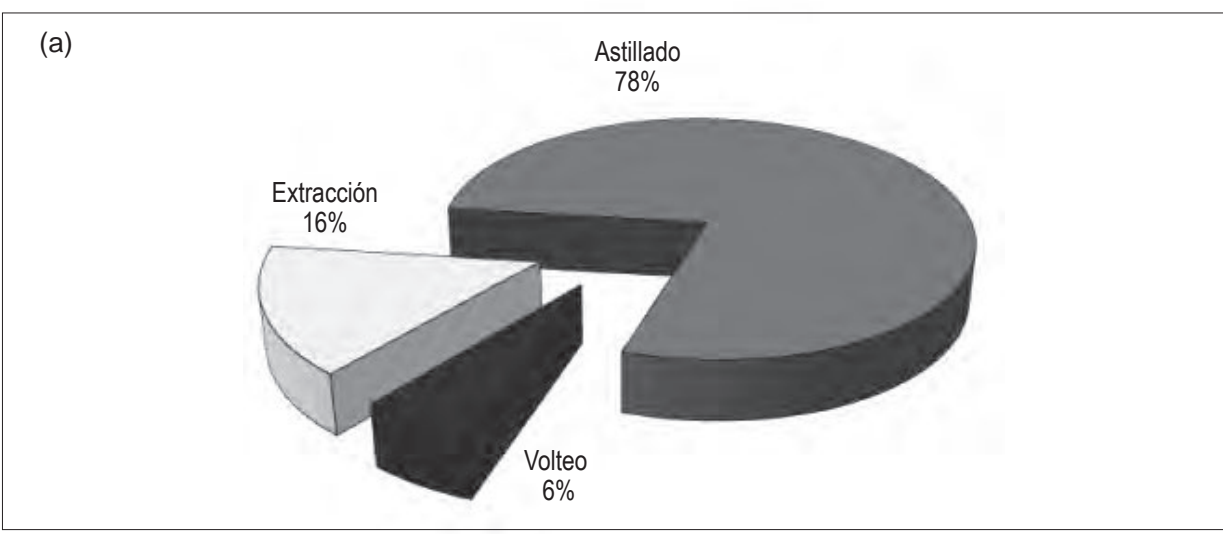

(b)

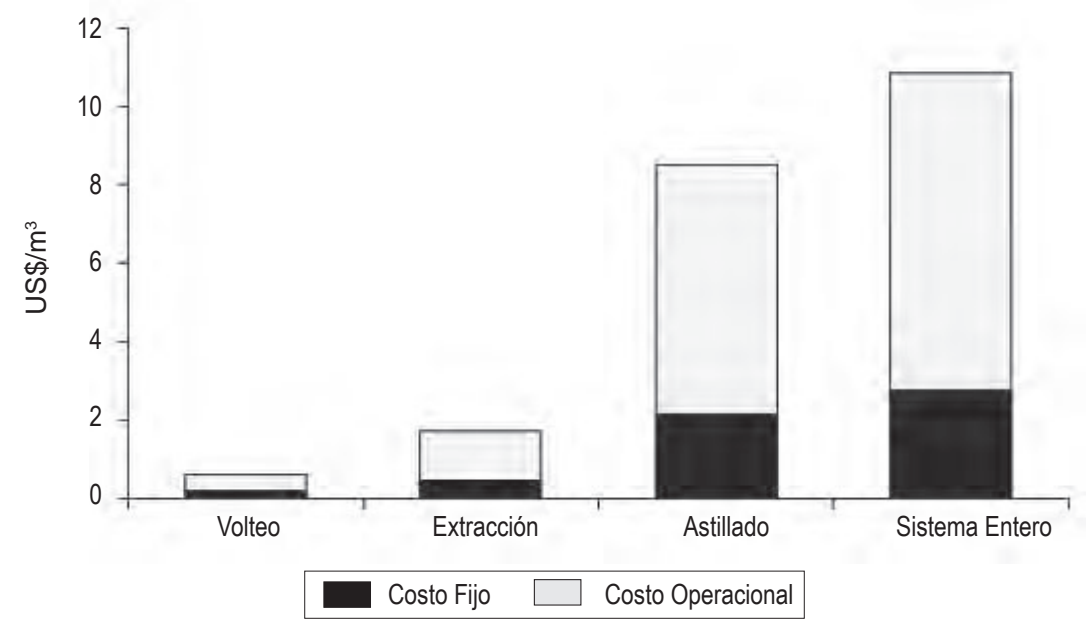

Figura 6. Costos totales por metro cúbico del sistema de cosecha y astillado segregados de acuerdo a subsistema (a), y de acuerdo al tipo de costo (b), costos fijos ( $\square$ ) y costos operacionales ( $\square$ ). 
totales y el restante $74 \%$ corresponde a costos operacionales (Figura 6b).

\section{DISCUSIÓN}

Las curvas obtenidas a partir de la variación paramétrica del DAP y la DE medios del rodal muestran el comportamiento de los costos del sistema por efecto de cambios en estas variables. En la Figura 4 se observa que al mantener la DE media del rodal fija y variar el DAP medio, el costo del sistema es afectado. Por ejemplo, un simple cambio en el diámetro medio del rodal de 10 a $15 \mathrm{~cm}$ representa una disminución en los costos de producción del $44 \%$. La DE media del rodal también afecta los costos del sistema pero en menor medida, de tal forma que al disminuir la DE media de 200 a $100 \mathrm{~m}$ se logra una disminución en los costos de producción del 3\% (Figura 5).

La aplicación construida con la plataforma iThink permitió evaluar y separar los costos por metro cúbico entre las actividades realizadas. De esta forma el análisis económico del sistema de cosecha puede ser efectuado con una mayor profundidad. En el caso mostrado en este estudio un DAP medio de rodal de $20 \mathrm{~cm}$ y una DE media de extracción de $200 \mathrm{~m}$ producen costos de US $\$ 0,63 / \mathrm{m}^{3}$ en el volteo, US $\$ 1,73 / \mathrm{m}^{3}$ en la extracción y US $\$ 8,50 / \mathrm{m}^{3}$ en el astillado.

\section{CONCLUSIONES}

Los elevados costos de este sistema de cosecha altamente mecanizado hacen necesario llevar a cabo una mejor planificación de las operaciones y de esta forma mantener óptima su capacidad de producción. La información proporcionada por la aplicación permite a la empresa forestal hacer un mejor uso de los equipos de cosecha y mejorar la rentabilidad del negocio.

La aplicación construida con la plataforma iThink mostró ser una herramienta versátil en el análisis de sistemas de cosecha, por lo que los resultados proporcionados conducen a un mejoramiento de las condiciones necesarias para el estudio económico de los mismos.
La información provista por las curvas de costo unitario podría ser utilizada fácilmente para calcular los costos que implicaría cosechar un rodal con determinadas características. Este es un primer paso para la realización de estudios económicos más profundos que contribuyan a establecer tarifas de pago más apropiadas teniendo en cuenta que el costo de cosecha varía de acuerdo a las características del rodal en el cual se realizará la cosecha.

\section{REFERENCIAS BIBLIOGRÁFICAS}

Arroyo, C. 2005. Evaluación técnica y modelamiento de procesador en cosecha de Pinus radiata en la X Región. Tesis de Ingeniería Forestal. Instituto de Manejo Forestal. Facultad de Ciencias Forestales. UACH. Valdivia, Chile. Pg. 31.

Barret, S. 2001. A Computer Simulation Model for Predicting the Impacts of Log Truck TurnTime on Timber Harvesting System Productivity. Tesis de Master of Science. Faculty of Virginia Polytechnic Institute and State University. Blacksburg, EU. Pg. 74.

Bradley, D., F. Biltonen \& S. Winsauer. 1976. A Computer Simulation of Full-Tree Field Chipping and Trucking. USDA Forest Service. North Central Forest Experiment Station. Pg. 19.

Carey P., A. Figueroa \& P. Valenzuela. 2006. Evaluación técnica de un sistema tradicional de cosecha en plantaciones de Eucalyptus globulus de corta rotación en Valdivia, Chile. Bosque 27 (3): 272-276.

Figueroa, A. 2004. Evaluación técnica y económica de sistema de cosecha tradicional de Eucalyptus sp. en la X Región. Tesis de Ingeniería Forestal. Instituto de Manejo Forestal. Facultad de Ciencias Forestales. UACH. Valdivia, Chile. Pg. 36.

Isee Systems, Inc. 2007. iThink v. 9.0 (trial). Lebanon, NH. EU.

Jaramillo, A. 2005. Evaluación técnica y económica de sistema de astillado en bosque de Eucalyptus sp. Tesis de Ingeniería Forestal. Instituto de Manejo Forestal. Facultad de Ciencias Forestales. UACH. Valdivia, Chile. Pg. 41. 
McDonagh, K. 2002. Systems Dynamics Simulation to Improve Timber Harvesting System Management. Tesis de Master of Science. Faculty of Virginia Polytechnic Institute and State University. Blacksburg, EU. Pg. 142.

McDonagh, K., R. Meller, R. Visser \& T. McDonald. 2004. Harvesting System Simulation Using a Systems Dynamic Model. Southern Journal of Applied Forestry 28 (2): 91-99.

Provoste, F. 2004. Descripción técnica y económica de un sistema de cosecha tradicional de
Eucalyptus sp. con la incorporación de descortezado mecánico en la X Región. Tesis de Ingeniería Forestal. Instituto de Manejo Forestal. Facultad de Ciencias Forestales. UACH. Valdivia, Chile. Pg. 46.

Winsauer, S. 1982. A Program and Documentation for Simulation of Grapple Skidders and a Whole-Tree Chipper. USDA Forest Service. North Central Forest Experiment Station. Pg. 42. 


\section{ANEXO}

Anexo 1. Costos del sistema de cosecha y astillado en bosque (US\$ del año 2007).

\begin{tabular}{|c|c|c|c|}
\hline ITEMS & $\begin{array}{c}\text { TigerCat } \\
720 \mathrm{C} \\
\text { Feller-Buncher }\end{array}$ & $\begin{array}{c}\text { TigerCat } \\
\text { 620C } \\
\text { Skidder Grapple }\end{array}$ & $\begin{array}{c}\text { Paterson Pacific } \\
\text { DDC } 5000 \mathrm{G} \\
\text { Whole Chipper }\end{array}$ \\
\hline \multicolumn{4}{|l|}{ COSTOS FIJOS } \\
\hline Precio de Compra (\$) & $220.000,00$ & $195.000,00$ & $720.000,00$ \\
\hline Valor de Reventa (\$) & $55.000,00$ & $55.000,00$ & $144.000,00$ \\
\hline Número de Equipos & 1 & 1 & 1 \\
\hline Vida Útil (Años) & 5 & 5 & 7 \\
\hline Horas Planificadas por Año (H/Año) & 4560 & 4560 & 4560 \\
\hline Porcentaje de Utilización (\%) & 100 & 100 & 100 \\
\hline Interés, Seguros, Licencias (\%) & 15 & 15 & 15 \\
\hline Depreciación (\$/H) & 7,24 & 6,14 & 18,05 \\
\hline Inversión Media Anual IMA (\$/Año) & $154.000,00$ & $139.000,00$ & $473.142,84$ \\
\hline Interés, Seguros, Licencias ISS (\$/H) & 5,07 & 4,57 & 15,56 \\
\hline Total Costos Fijos $(\$ / H)$ & 12,3 & 10,71 & 33,61 \\
\hline \multicolumn{4}{|l|}{ COSTOS OPERACIONALES } \\
\hline Promedio de Consumo de Combustible (L/H) & 28 & 30 & 80 \\
\hline Costo Unitario del Combustible (\$/L) & 1,15 & 1,15 & 1,15 \\
\hline Consumo de Lubricantes (\% Combustible) & 30 & 30 & 30 \\
\hline Mantenimiento y Reparaciones (\% Depreciación) & 60 & 60 & 60 \\
\hline Costo Horario de Combustible $(\$ / H)$ & 32,2 & 34,5 & 92 \\
\hline Costo Horario de Lubricantes $(\$ / H)$ & 9,66 & 10,35 & 27,6 \\
\hline Costo Mantenimiento y Reparaciones $(\$ / H)$ & 4,34 & 3,68 & 10,83 \\
\hline Costo de Accesorios - Neumáticos, 0tros $(\$ / H)$ & 2,33 & 2,33 & 0,66 \\
\hline Total Costos Operacionales $(\$ / H)$ & 48,54 & 50,87 & 131,09 \\
\hline \multicolumn{4}{|l|}{ MANO DE OBRA } \\
\hline Costo Mano de Obra $(\$ / H)$ & 6,37 & 6,37 & 7,82 \\
\hline Total Costos Mano de Obra $(\$ / H)$ & 6,37 & 6,37 & 7,82 \\
\hline Gran Total (\$/H) & 67,21 & 67,95 & 172,52 \\
\hline
\end{tabular}

